\title{
Correction to: Interaction effects of energy efficiency policies: a review
}

\author{
Catharina Wiese (D) - Anders Larsen • Lise-Lotte Pade
}

Published online: 6 July 2018

(C) Springer Nature B.V. 2018

\section{Correction to: Energy Efficiency https://doi.org/10.1007/s12053-018- 9659-z}

In the original publication, 8 paragraphs under subsection Interaction cases were incorrectly set as footnotes of Table 2. The paragraphs are:

(1) Boonekamp (2006) and Braathen (2007) classify the combination of a performance standard with an energy tax as mitigating. Boonekamp (2006) argues that the target group of a standard, which sets a high and legally binding requirement, has to fulfil this standard, while a tax would not lead to the implementation of additional measures to increase energy efficiency. Thus, he points at the prescriptive policy mechanism of performance standards, which force the energy end-user to save energy,

The online version of the original article can be found at https://doi.org/10.1007/s12053-018-9659-z

C. Wiese $(\bowtie) \cdot$ L.-L. Pade

Department of Management Engineering, Systems Analysis Division, Technical University of Denmark, Produktionstorvet Building 426, 2800 Kongens Lyngby, Denmark e-mail: catwi@dtu.dk

\section{A. Larsen}

Department of Social Sciences and Business, Roskilde University, Universitetsvej 1, 4000 Roskilde, Denmark thus no further motivation is needed, and defines this mechanism as the reason for the mitigating interaction. Braathen (2007) takes this combination as an example for mitigating interaction effects, which hinder the effective and efficient functioning of both instruments and cause redundancies and unnecessary administrative costs.

(2) Furthermore, Boonekamp (2006) assesses that the combination of an energy tax with financial incentives, i.e. different subsidy schemes, can bemitigating or reinforcing depending on the specific application of the subsidy. On the one side, Boonekamp (2006) discusses that both instruments target the motivation of energy end-users to invest in energy saving options and together they provide too much motivation, i.e. only one instrument would have led to the same investment decision. On the other side, he argues that a subsidy, which specificallymotivates saving options that are not yet established and still expensive, can have a reinforcing interaction with an energy tax. In that case, consumers would not have chosen to implement these saving options onlymotivated by a tax. Thus, the target of a subsidy scheme, i.e. proven or not yet established saving options, determines the interaction outcome.

(3) Rosenow et al. (2016) highlight that a tax on energy has a reinforcing interaction with all other instruments they include in their analysis. They argue that the direct price effect of a tax generally increases the incentive and motivation of end-users to invest in energy efficient technology and reduce 
energy consumption, i.e. to use financial incentives, implement regulation or join voluntary agreements. Thus, the price mechanism of a tax strengthens the functionality of other instruments. Furthermore, Child et al. (2008) classify the combination of an energy tax with a TWCscheme as reinforcing and reason that with a tax as the single instrument, end-usersmay choose to pay the tax when it is expensive to reduce consumption. The combination with a white certificate scheme, which implies the provision of financial incentives, increases the amount of affordable energy saving options and the final energy saving effect.

(4) Assessing the combination of EEOs with financial incentives, Rosenow et al. (2016) point out that the obligation scheme implies a capped saving level, which entails that financial incentives on top of the scheme would not achieve additional savings, and classify this combination as mitigating. Thus, similar to the policy mechanism of performance standards in (1), the predefined energy saving target of EEOs limits the effectiveness of additional financial incentives. On the contrary, Child et al. (2008) conclude that the combination of TWCs with financial incentives is reinforcing, because the increase in total compensation for energy efficiency investment (increase in financial support available) accelerates technology diffusion of energy efficient equipment. However, they also consider that this combination may be an unnecessary use of resources once a technology becomes standard in the market.

(5) Rosenow et al. (2015) classify the combination of voluntary agreements with EEOs as mitigating and argue that the obligation scheme sets a certain energy saving target, so that a voluntary agreement, which targets the same sector and aims at a similar saving level, would not generate additional savings. Child et al. (2008), when assessing the combination of TWCs and voluntary agreements, highlight the challenge of the measurement and verification of savings, which the voluntary agreement scheme achieves, as being eligible to count as a saving certificate.

(6) On one side, the combination of performance standards with financial incentives is mitigating, when the financial support finances investments that are required by the performance standard, as Rosenow et al. (2015) evaluate. In that case, the legally binding target of the standard entails that additional financial incentives do not increase effectiveness, but the number of free-riders, here defined as agents that make use of a subsidy, although they have to do a certain investment to fulfil the standard. On the other side, the bigEE project argues that financial incentives in combination with performance standards are important to trigger energy efficiency investments, especially in the presence of high financing barriers. Thus, this combination of policies ensures a broad market introduction of energy efficient products and finally enables policy makers to tighten the standard and achieve higher future efficiency levels.

(7) Furthermore, Rosenow et al. (2016) discuss that two instruments, which both provide a financial incentive for energy efficiency investments, cause a mitigating interaction, when the recipient had made the same investments in the presence of only one of the two instruments. In that case, the benefit recipient is overpaid.

(8) All studies categorise the provision of information, especially via labelling schemes, as mutually reinforcing. Thus, providing information supports the effectiveness of all other instruments and vice versa. E.g. Braathen (2007) illustrates that a label increases the awareness of consumers and therefore their responsiveness to energy prices. This effect finally increases the effectiveness of a priceincreasing tax on energy. Moreover, consumers may be more attentive to a label due to a tax. Thus, the policy mechanism of information provision to increase the awareness of end-users towards their energy consumption determines the mutually reinforcing interaction with other instruments. Yet, Braathen (2007) also mentions the exceptional case that the provision of too much information, e.g. due to the implementation of various different labelling schemes, may cause confusion and a mitigating combined effect. Considering the combination of information provision (in particular building certificates) with financial incentives, Child et al. (2008) furthermore point out that information provision may increase the free-rider problem. I.e., the increase in awareness entails that more consumers would increase their energy efficiency investments without financial incentives, but are still able to receive them.

The original publication was corrected. 